\title{
Aspectos Morfológicos e Relação Estrutura- Propriedades de Poliestireno de Alto Impacto
}

\author{
Vinícius G. Grassi e Maria Madalena C. Forte \\ Laboratório de Materiais Poliméricos, Departamento de Materiais, Escola de Engenharia, UFRGS
}

\author{
Marcus F. Dal Pizzol \\ Pesquisa \& Desenvolvimento, Innova S.A.
}

Resumo: A tenacificação da matriz vítrea de poliestireno (PS) pela adição de borracha polibutadiênica, que tem como consequiência um aumento de sua resistência ao impacto, origina um polímero com características singulares, o Poliestireno de Alto Impacto (HIPS). Durante a polimerização in situ de estireno em uma solução de borracha, ocorre a formação de cadeias graftizadas de poliestireno no polibutadieno, as quais aumentam a interação interfacial borracha-PS. O HIPS é um material com ampla aplicação na indústria de embalagens e, principalmente, em gabinetes de refrigeradores, a chamada linha branca. O objetivo deste artigo é destacar e discutir as características estruturais e morfológicas do HIPS e a implicação destas na determinação de suas propriedades. Também foram revisadas as técnicas comumente utilizadas na caracterização morfológica e estrutural do HIPS.

Palavras-chave: Poliestireno de alto impacto, HIPS, graftização, resistência química, fase gel.

\section{Morphologic Aspects and Structure-Properties Relations of High Impact Polystyrene}

\begin{abstract}
Toughening of glassy polystyrene (PS) matrix by polybutadiene rubber addition mainly increases its impact resistance. The rubber modified polymer, known as High Impact Polystyrene (HIPS), has unique features. During the in situ styrene polymerization in a rubber solution grafting of polystyrene chains occurs onto polybutadiene molecules and magnifies the interaction at the rubber-PS interface. The HIPS is a material that has a large application in the packaging industry and refrigerator cabinets. The aim of this paper is to point out and discuss some of the structural and morphological characteristics of HIPS and its correlation with general properties. The standard techniques applied in the characterization of the structure and morphology of HIPS were revised.
\end{abstract}

Keywords: High impact polystyrene, HIPS, grafting, chemical resistance, gel phase

\section{Introdução}

A incorporação de uma segunda fase elastomérica em uma matriz vítrea de um polímero tem como principal objetivo o aumento de sua tenacidade, ou seja, de sua resistência ao impacto ${ }^{[1,2]}$. O poliestireno (PS) à temperatura ambiente é um polímero vítreo e apresenta baixa absorção de energia sob impacto devido a ausência de mobilidade local de segmentos de cadeia, uma vez que a sua $\mathrm{T}_{\mathrm{g}}$ ocorre entre 90 e $100^{\circ} \mathrm{C}$, temperatura a partir da qual ocorrerem movimentos de segmentos de cadeia responsáveis pela dissipação de energia. A utilização de borracha no processo de polimerização do estireno leva à obtenção de uma mistura polimérica in situ, com domínios discretos de fase borrachosa dispersos em uma matriz contínua de PS. A polimerização borracha-estireno resulta em um produto com propriedades mecânicas sob impacto superiores, o Poliestireno de Alto Impacto (HIPS), quando comparado ao PS e mesmo a blendas de PS e borracha. Esse aperfeiçoamento deve-se principalmente à introdução de um componente amorfo flexível

Autor para correspondência: Madalena C. Forte, Laboratório de Materiais Poliméricos, Departamento de Materiais, Escola de Engenharia, UFRGS, Av. Osvaldo Aranha 99, CEP: 90035-190, Porto Alegre, RS. E-mail: mmcforte@ufrgs.br 
$\left(\mathrm{T}_{\mathrm{g}} \leq-40^{\circ} \mathrm{C}\right)$ na matriz rígida de PS e: (1) ao grau de ligações cruzadas da borracha, (2) à interação química entre cadeias de PS formadas e cadeias da borracha, com aumento da adesão interfacial entre as fases PSborracha, e (3) às oclusões de fase contínua de PS no interior das partículas de borracha, com aumento da fração volumétrica desta última. A morfologia é um dos principais fatores responsáveis pelo elevado desempenho mecânico do HIPS. Ainda que o HIPS possa ser produzido por polimerização em emulsão, o processo comercial atualmente utilizado é o de polimerização em massa, no qual a borracha é solubilizada pelo monômero estireno, também podendo ser considerado um processo em solução. A reação de polimerização é feita através do uso de iniciadores, geralmente peróxido de benzoíla. Em HIPS comerciais, o tamanho e a distribuição de tamanhos das partículas de borracha são controlados pela taxa de cisalhamento do processo, durante e após a inversão de fases, isto é, quando o PS passa a ser a fase contínua e a borracha a fase dispersa, pelas viscosidades relativas das fases e pela tensão interfacial entre essas fase $\mathrm{s}^{[3,4]}$. O primeiro estudo documentado sobre a tenacificação do PS data de 1927, sendo uma patente de processo de produção de PS tenacificado a partir da polimerização de estireno em uma solução de borracha no próprio monômero ${ }^{[5]}$. $\mathrm{O}$ processo não obteve sucesso comercial, uma vez que o produto formado era reticulado, não ocorrendo a inversão de fases. Desenvolvimentos posteriores feitos pela empresa Dow Chemical Company, nos anos 40 e 50, resultaram em produtos comerciais de PS e copolímeros estireno-butadieno (SBR) obtidos em processo em emulsão, os quais passaram a ser chamados de Poliestireno de Alto Impacto (HIPS). A partir de 1960, as borrachas do tipo SBR preferencialmente incorporadas ao PS passaram a ser substituídas por polibutadieno $(\mathrm{PB})^{[6]}$, devido a obtenção de HIPS com propriedades mecânicas sob impacto superiores, especialmente pela ocorrência de uma maior graftização e entrecruzamento da borracha .

O teor de borracha em resinas de HIPS comerciais obtidas em processo de polimerização em massa está limitado a $14 \%$ em peso devido à alta viscosidade do meio, sendo o conteúdo de borracha normalmente inferior a $10 \%$. A limitação do volume da partícula de borracha devido à restrição do teor da mesma é compensada pela oclusão de domínios de PS no interior dessas partículas, os quais podem aumentar a fração volumétrica da fase tenacificadora de 10 a $40 \%{ }^{[3]}$.
O objetivo deste artigo é apresentar e discutir alguns parâmetros importantes da tenacificação do PS, como características estruturais e morfológicas, mecanismos de tenacificação e propriedades mais importantes, bem como revisar as técnicas usuais de caracterização do HIPS. A correlação entre estrutura-propriedade foi feita com bases em dados de literatura e dados obtidos em experimentos de laboratório de diversas amostras comerciais produzidas por polimerização em massa.

\section{Características do HIPS}

\section{Graftização e Entrecruzamento}

A graftização do PB é iniciada pela abstração de hidrogênios alílicos da sua cadeia macromolecular por radicais livres gerados no meio, seguida da adição de um macrorradical de PS em crescimento na mesma, conforme representado esquematicamente na Figura 1.

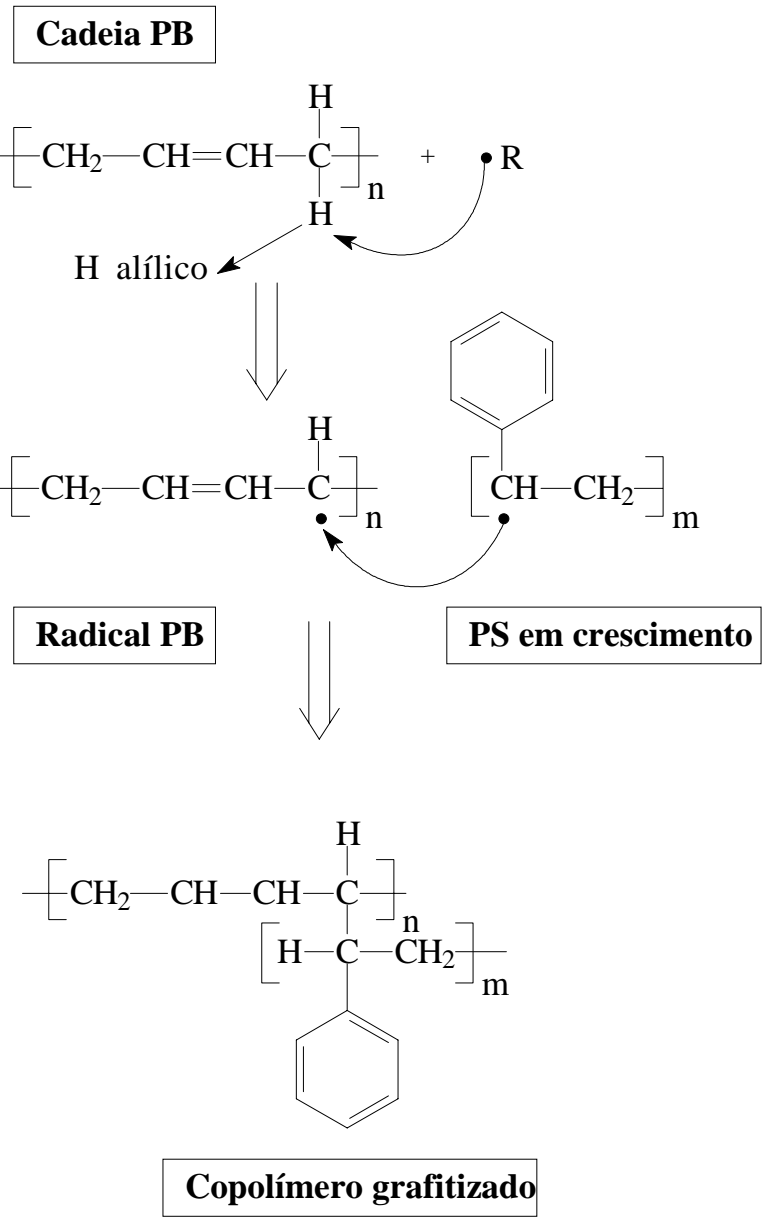

Figura 1. Mecanismo de formação do copolímero grafitzado PB-PS, onde $\mathrm{R} \bullet=$ qualquer radical formado no meio reacional 
$\mathrm{Na}$ ausência do monômero estireno, somente o aquecimento não induz à formação de ligações cruzadas (entrecruzamento) no PB, o que demonstra que as duplas ligações presentes na cadeia do PB não participam diretamente da graftização ou da formação de ligações cruzadas durante a polimerização térmica do estireno na formação do HIPS. A adição de um macrorradical de PS na cadeia de PB leva principalmente à formação de PB graftizado, com estrutura em "T" em detrimento de outras estruturas. A incorporação de uma ou mais cadeias de PS na cadeia de PB pode ocorrer através de diferentes possibilidades de combinação de segmentos ou blocos radicais de PS com os macrorradicais de PB, conforme ilustrado na Figura 2 c e d, observando-se a formação de copolímeros graftizados com estrutura em "T" e "H", respectivamente. Pode ainda haver a combinação entre macrorradicais de PS e de PB, com formação de PS homopolímero (Figura 2 a) e PB com ligações cruzadas (Figura 2 b) ${ }^{[7]}$.

O PS homopolímero é produzido essencialmente através do mecanismo de terminação por combinação de macrorradicais de PS, entre outros, enquanto os macrorradicais de $\mathrm{PB}$ podem reagir entre si, levando à formação de um PB com diferentes graus de entrecruzamento. O copolímero PB-PS graftizado pode ser formado pela combinação entre cadeias radicais de PB e uma ou mais macrorradicais de PS, caracterizando a graftização em "T", responsáveis pela melhor adesão superficial entre a matriz de PS e

a)

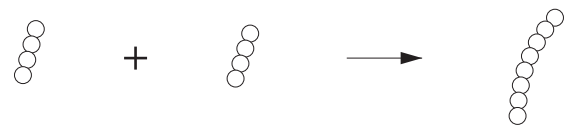

b)

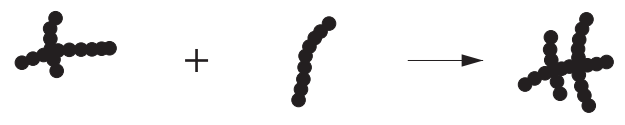

c)

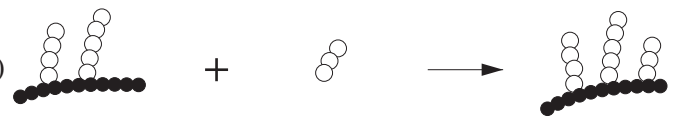

d)

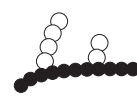

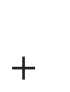

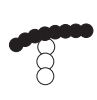

a partícula borrachosa no HIPS. O PB graftizado ainda pode ser formado pela combinação de duas cadeias de PB graftizadas com radicais de PS em crescimento, levando à formação de graftização em "H". Essa combinação também pode ser considerada como uma espécie de entrecruzamento que envolve as cadeias de PS e aumenta a rigidez do sistema. As ligações cruzadas proporcionam estabilidade morfológica às partículas de borracha. $\mathrm{O}$ grau de entrecruzamento não deve ser muito alto de forma a não comprometer a propriedade de resistência ao impacto do HIPS. A formação do PS graftizado é detectada quando a conversão do estireno alcança $2 \%{ }^{[8]}$. A formação do PS homopolímero ocorre durante toda a polimerização. Em média, cerca de $80 \%$ do estireno é convertido em PS homopolímero e $20 \%$ é graftizado nas cadeias de PB, em outras palavras, a formação do PS é quatro vezes mais rápida que a graftização. Além disso, as cadeias de PS graftizadas nas de PB são menores que as de PS homopolímero. Tanto a graftização quanto o entrecruzamento da borracha aumentam com a temperatura de polimerização e conversão. Com o aumento da conversão, a graftização e, conseqüentemente, o entrecruzamento da borracha são favorecidos em relação à hopolimerização do estireno simplesmente porque os sítios de graftização nas cadeias de PB tornam-se progressivamente mais disponíveis em relação ao monômero estireno.

A eficiência da borracha na tenacificação do HIPS é maior com o aumento da graftização e a redução do grau de entrecruzamento ${ }^{[9]}$. A melhor maneira de maximizar a graftização e minimizar o entrecruzamento é manter a polimerização em alta conversão à baixa temperatura. Para uma mesma fração volumétrica da fase tenacificadora, verifica-se que, quanto menor o grau de entrecruzamento, maior será a resistência ao impacto do HIPS. Um grau de entrecruzamento baixo permite que as partículas de borracha sejam mais deformáveis e hábeis no processo de relaxação, absorvendo melhor a energia do impacto. Quanto ao índice de graftização, verifica-se que, quanto maior for este maior será a interação entre as fases vítrea e borrachosa e, portanto, maior será a resistência do material ao impacto. A Figura 3 ilustra esquematicamente o tipo de interação ao nível molecular que ocorre entre a fase borrachosa e a fase vítrea do PS. Esta será tanto maior e melhor quanto maior for o número de moléculas de PB graftizado,
Figura 2. Possíveis combinações de macrorradicais na obtenção de: (a) PS livre; (b) PB reticulado; (c) copolímero graftizado em "T" e (d) copolímero graftizado em "H", onde $\mathrm{O}=$ unidade estirênica, $\bullet=$ unidade butadiênica e $\bigcirc$ = sítio de graftização 


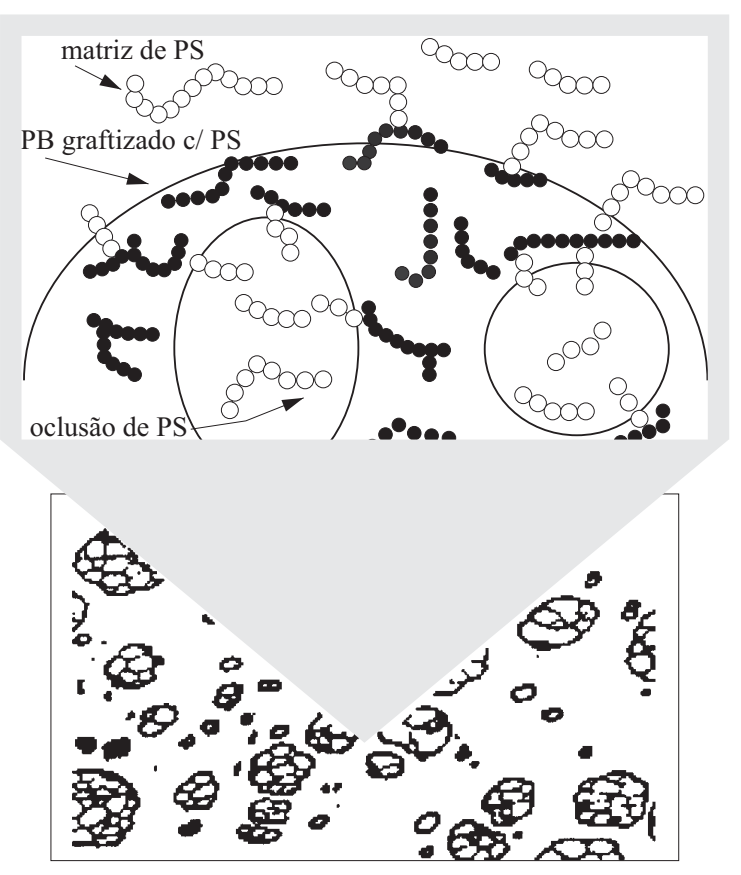

Figura 3. Interface PS-borracha em HIPS com morfologia tipo salami, onde: $\mathrm{O}=$ unidade estirênica, $\boldsymbol{Q}=$ unidade butadiênica e $\bullet \mathbf{O}=$ sitio de graftização

as quais se dispõe na interface entre as duas fases de maneira que os blocos de PS ficam na matriz ou oclusões de PS, enquanto a cadeia do PB graftizado fica na fase borrachosa. Desse modo, ocorre uma ligação física das fases rígidas e flexíveis, além da ligação covalente entre PS e PB no sítio de graftização. Esse tipo de interação inibe a separação das fases e, conseqüentemente, a formação de microfissuras e fratura do material.

\section{Morfologia}

As diferenças estruturais e morfológicas entre resinas de HIPS resultam de diferenças nos processos de produção e no tipo e teor de borracha ${ }^{[10,11]}$. Existe uma grande variedade de tipos ou grades de HIPS disponíveis comercialmente. Os mais comuns possuem tamanho médio de partícula de borracha de até $5 \mu \mathrm{m}$ de diâmetro e morfologia do tipo salami, conforme mostrado na Figura 4. Essa morfologia consiste de partículas de diferentes tamanhos, com subinclusões de PS envoltos por uma membrana de borracha, dispersas numa matriz de PS e é típica de HIPS obtido em processo de polimerização em massa.

A morfologia do HIPS obtido através de processo de polimerização em emulsão geralmente apresenta partículas de borracha esféricas, não-desejáveis

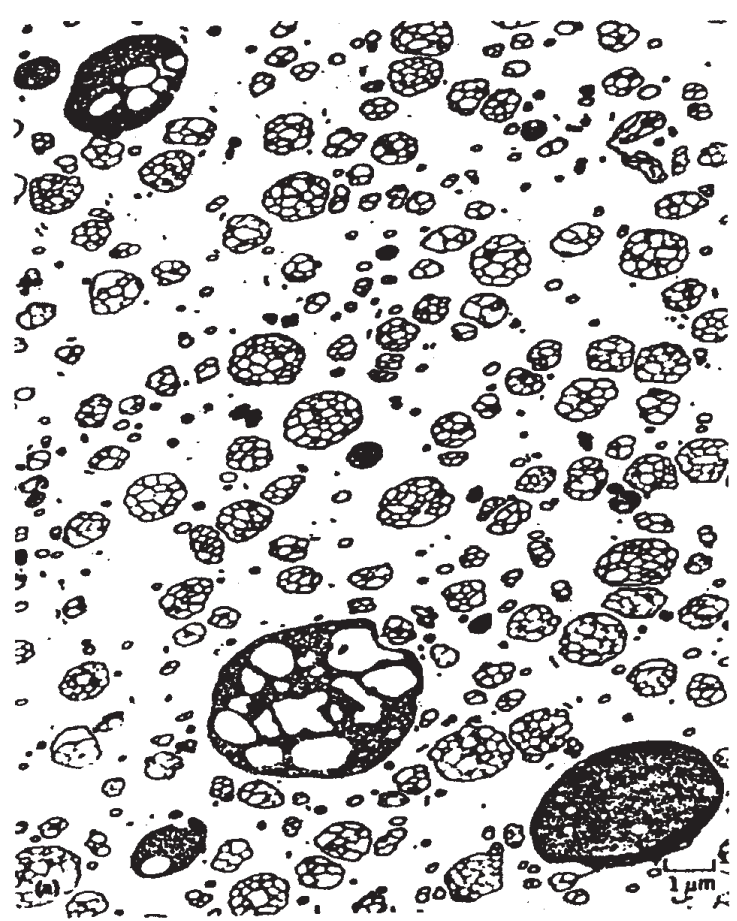

Figura 4. Imagem MET de HIPS produzido por polimerização em massa ou solução. Morfologia tipo salami ${ }^{[3]}$. A parte escura (fase dispersa) identifica a borracha e a parte clara (fase contínua e inclusões) o poliestireno.

por comprometer a transparência, e partículas com estrutura do tipo core shell, isto é, partículas compostas por um núcleo de PS (core) revestido por uma membrana de PB (shell), conforme mostrado na Figura 5. Essa morfologia não afeta significativamente a transparência do HIPS, sendo uma resina semitransparente utilizada na fabricação de embalagens. As partículas de PS recobertas por borracha são da ordem de 0,2 $\mu \mathrm{m}$ de diâmetro. O HIPS com tamanho

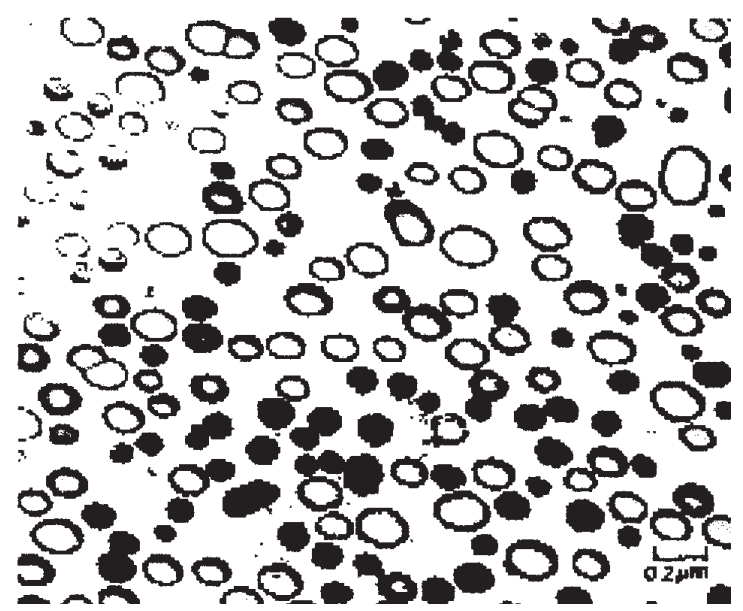

Figura 5. Imagem MET de HIPS produzido por polimerização em emulsão. Morfologia tipo core shell. A parte escura da fase dispersa identifica a borracha (shell) e a parte clara das inclusões o poliestireno (core) 


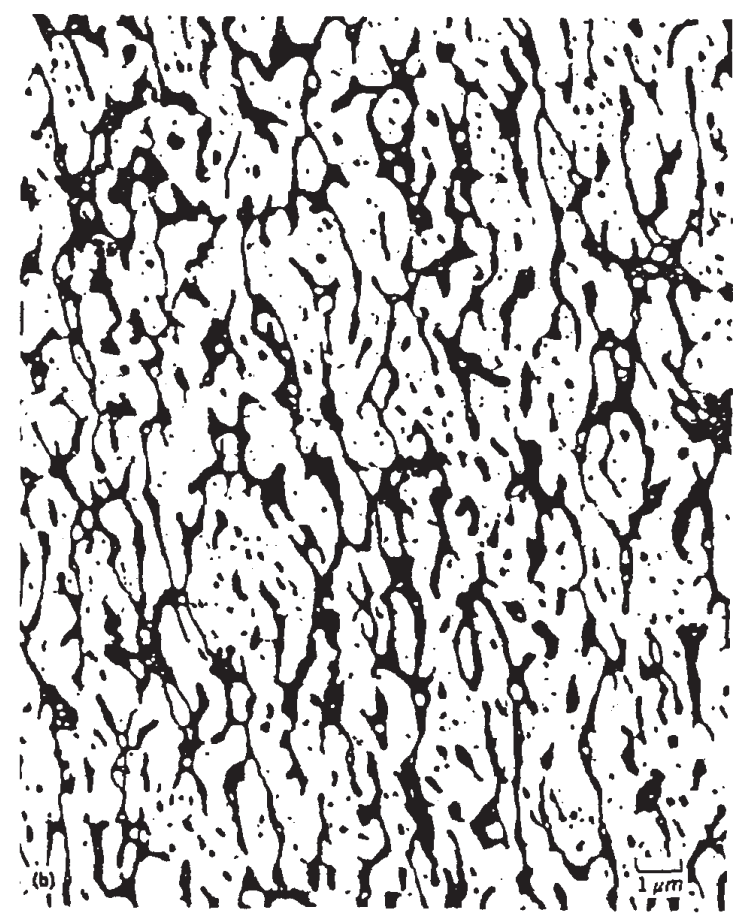

Figura 6. Imagem MET de uma blenda pós-reator de SBR e PS ${ }^{[3]}$

de partícula de borracha dessa ordem não apresenta a mesma tenacidade à fratura que o HIPS com morfologia tipo salami e apresenta uma resistência ao impacto apenas levemente superior àquela do PS homopolímero.

A Figura 6 mostra a morfologia típica de uma mistura pós reator de PS homopolímero e borracha tipo SBR (estireno-butadieno). A morfologia da mistura PS/SBR difere substancialmente daquela do HIPS, apresentando domínios de borracha dispersos no PS sem as oclusões características do HIPS com morfologia tipo salami ou core-shell. No caso da mistura não existe o mesmo tipo de interação interfacial entre as fases de PS e borracha.

\section{Mecanismo de Tenacificação}

Os mecanismos envolvidos na tenacificação do HIPS incluem essencialmente o microfissuramento (crazing) e a cavitação ${ }^{[,]}$. A presença de uma segunda fase tenacificadora na matriz de PS pode modificar significativamente o seu comportamento tensão $\mathrm{x}$ deformação, levando à ocorrência de modos de microdeformação com maior consumo de energia. Assim, a concentração, a dimensão, a morfologia e as propriedades da fase tenacificadora são extremamente relevantes no desempenho do material.

O mecanismo de microfissuramento é, sem dúvi- da, o principal responsável pela absorção e dissipação de energia de impacto no HIPS. A Figura 7 mostra esquematicamente o que acontece quando o HIPS é submetido a uma energia de impacto. De uma maneira geral, existe uma concordância no sentido de que as partículas de borracha absorvem a energia de impacto porque podem atuar da seguinte forma: (1) seriam iniciadores ou nucleadores de microfissuras e (2) agiriam como terminadores de microfissuras, evitando a transformação rápida das microfissuras em fratura $^{[14,15]}$.

Quando o HIPS é submetido a uma energia de impacto, parte dessa energia é dissipada pela borracha sob a forma de calor, pela vibração térmica de seus átomos e pelos movimentos de relaxação de segmentos da macromolécula. A energia restante é dissipada na formação de diversas microfissuras na interface da matriz de PS com a borracha. Essas microfissuras irão propagar-se até encontrar uma nova partícula de borracha na qual a energia irá novamente ser dissipada sob forma de calor e novas microfissuras poderão ser nucleadas, num processo de redistribuição da energia de impacto. O mecanismo de microfissuramento pode ser explicado em termos de propagação de microporos através da instabilidade de meniscos ou fibrilas que os separam. Como fatores que aumentam a formação de microfissuras, e portanto a absorção de energia de impacto, estão a boa adesão borracha-matriz, o correto tamanho das partículas ${ }^{[16,17]}$, o alto teor e a baixa $\mathrm{Tg}$ da borracha, a morfologia esférica das partículas tenacificadoras e o alto coeficiente de expansão térmica da borracha. A massa molar da matriz de PS tem efeito desprezível na formação de microfissuras, mas uma alta massa molar estabiliza as microfissuras uma vez que estas sejam formadas. O diâmetro médio das partículas de borracha tem influência significativa no mecanismo de microfissuramento. Partículas pequenas, com tamanho menor que $1 \mu \mathrm{m}$, são menos eficiente na

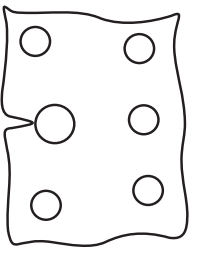

energia de impacto

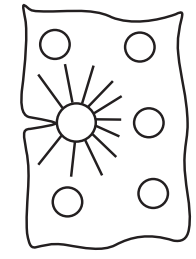

iniciação e crescimento das microfissuras

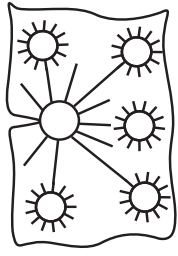

terminação e iniciação de novas microfissuras
Figura 7. Mecanismo de absorção de impacto do HIPS através do microfissuramento 
tenacificação do que partículas maiores $(2-5 \mu \mathrm{m})$ para um mesmo teor de borracha ${ }^{[18]}$.

A natureza da morfologia interna das partículas de borracha também tem grande importância no processo de microfissuramento. A presença de algumas pequenas subinclusões de PS permite que ocorra a fibrilação, causada pela orientação das cadeias de PS no sentido perpendicular à tensão aplicada e geradora de microporos. A elongação do material ao longo da direção da tensão aplicada é acompanhada por uma significativa contração da fase borracha na direção equatorial, gerando poros grandes na interface borracha-matriz. Como os argumentos em relação à mecânica de fratura indicam que os poros grandes são mais danosos que os poros pequenos, deve-se evitar a formação de partículas pequenas compostas exclusivamente de borracha, ou seja, sem as oclusões de PS, as quais podem dar origem a poros grandes.

A partir das questões consideradas, tanto a iniciação quanto a terminação de microfissuras pelas partículas de borracha podem ocorrer de maneira satisfatória: (1) minimizando-se o número de partículas sólidas de todos os tamanhos (compostas exclusivamente de borracha, sem oclusões de PS), (2) evitando-se a formação de partículas de borracha menores que um diâmetro crítico para a nucleação de microfissuras e (3) favorecendo-se a formação de partículas maiores que o diâmetro crítico (para maximizar o total de partículas que iniciam microfissuras), preferencialmente com um grande número de pequenas oclusões de PS.

Nos polímeros tenacificados com borracha, a geração de poros pode ocorrer na matriz ou no interior das partículas de borracha, ocasionando o que se chama de cavitação da borracha. A cavitação ocorre devido a uma expansão elástica do precursor da cavidade, permitindo um alívio da tensão hidrostática do material e iniciando um escoamento por cisalhamento da matriz ${ }^{[19]}$. Entretanto, o mecanismo de cavitação ocorre no HIPS sem que haja o escoamento da matriz ${ }^{[20]}$.

Fase Gel e Tamanho e Distribuição de Tamanhos de Partícula de Borracha

A fase gel representa a fração insolúvel do HIPS em tolueno, segundo a técnica de separação de fases de Ruffing ${ }^{[33]}$, sendo composta pelo PB nãograftizado, pelo $\mathrm{PB}$ entrecruzado, pelo PB graftizado (localizado na interface PS-PB) e pelo PS ocluído no interior das partículas de borracha. A elevação da resistência ao impacto com o conteúdo de fase gel está relacionada com a maior deformação plástica da matriz modificada pela borracha ${ }^{[21,22]}$.

O tamanho ideal das partículas da fase gel para uma boa tenacificação do HIPS produzido em processo em massa está na faixa de 1-4 $\mu \mathrm{m}$. A elevação da resistência ao impacto com o conteúdo de fase gel varia linearmente somente para partículas com diâmetro de $2-3 \mu \mathrm{m}$. Para partículas menores $(1,3-2 \mu \mathrm{m})$, o aumento da resistência ao impacto com o conteúdo de fase gel é progressivamente maior. O inverso ocorre no caso de partículas maiores (3-4 $\mu \mathrm{m}$ de diâmetro), nas quais há um aumento da resistência ao impacto menos pronunciado. Para partículas com diâmetro menor que $1 \mu \mathrm{m}$, a resistência ao impacto do material é muito pequena ${ }^{[23,24]}$.

O tipo de agitação do meio de polimerização e a quantidade e tipo de borracha têm efeito significativo no tamanho e na distribuição de tamanhos das partículas. $\mathrm{O}$ aumento da taxa de agitação durante a polimerização implica uma diminuição no tamanho das partículas formadas em função do aumento do cisalhamento da borracha. Com a diminuição do tamanho da partícula de borracha, não ocorre coalescência, provavelmente devido à estabilidade morfológica conferida pelo entrecruzamento parcial da borracha.

Uma distribuição bimodal de partículas de borracha com uma população de partículas pequenas e outra de partículas maiores dispersas na matriz mostrou ser mais efetiva na resistência ao impacto do HIPS que a distribuição monomodal para um mesmo teor de borracha. A principal explicação para esse comportamento é que as partículas pequenas $(<1 \mu \mathrm{m})$ iniciam as microfissuras, ao passo que as maiores $(>1 \mu \mathrm{m})$ terminam e controlam a propagação das mesmas $^{[25]}$.

\section{Propriedades do HIPS}

Certas aplicações características das resinas de HIPS requerem propriedades superiores àquelas apresentadas pelo PS convencional, como maior resistência ao impacto e melhor ductilidade. Um balanço da resistência ao escoamento e da resistência térmica é necessário, sem que haja a perda do brilho do material. As propriedades requeridas para o HIPS, principalmente a resistência ao impacto, são dependentes de fatores como teor e tipo de borracha, tamanho e 
morfologia das partículas de borracha, volume da fase tenacificadora, grau de entrecruzamento e graftização, massa molecular e distribuição de massas molares da fase PS, comportamento viscoelástico da borracha e grau de adesão entre borracha e matriz. Como as variáveis que afetam as propriedades não são independentes, a otimização de uma propriedade pode ser obtida em detrimento de outra ${ }^{[3,26]}$.

A Figura 8 mostra um quadro resumo da variação de algumas propriedades do HIPS em relação a características estruturais da fase gel ou borracha, como teor e grau de entrecruzamento de PB, graftização, tamanho de partícula, e em relação à massa molar da matriz ${ }^{[3]}$. Como se pode notar, a tenacidade do HIPS é a propriedade que mais varia em função das modificações da fase borracha, apresentando um acréscimo significativo, com exceção do aumento do número de ligações cruzadas. O módulo elástico tende a diminuir somente quando há um aumento do teor e tamanho da partícula de borracha, o que diminui a rigidez do material. A temperatura de deflexão térmica não sofre influência com a variação da maioria das variáveis, mas somente com o aumento do teor de borracha, afinal é uma propriedade que depende mais significativamente da fase contínua, que é responsável pela rigidez do material. O brilho tende a diminuir com a introdução de borracha, com exceção do caso em que o número de ligações cruzadas aumenta. Essa propriedade é geralmente melhorada pelo uso de partículas pequenas de borracha $(<1 \mu \mathrm{m})$. O índice de fluidez do HIPS diminui sensivelmente com o aumento da massa molar da matriz e, por outro lado, aumenta à medida que o tamanho da partícula de borracha aumenta. O ESCR (environmental stress cracking resistance) aumenta sempre que ocorre um incremento no valor das características estruturais avaliadas.

A propriedade de resistência química do HIPS é extremamente importante em função de sua larga aplicação na indústria de embalagens e na fabricação de gabinetes de refrigeradores. As embalagens de HIPS podem sofrer ataque químico dos alimentos e apresentar perda de suas propriedades fisico-mecânicas. No caso de refrigeradores, o gabinete em HIPS tem contato com a espuma de poliuretana responsável pelo isolamento térmico do gabinete e, portanto, pode sofrer ataque do agente de expansão da espuma, normalmente gás freon ou ciclopentano ${ }^{[27]}$. Um dos efeitos do contato do HIPS com determinadas subs-

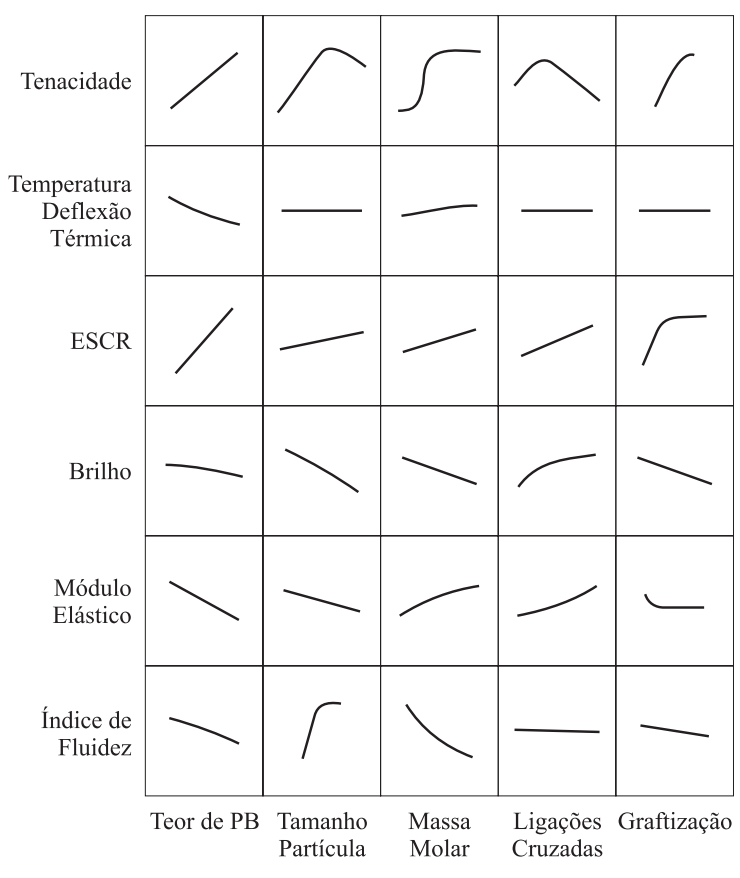

Figura 8. Quadro-resumo das variações de algumas propriedades do HIPS versus caracterísiticas estruturais típicas

tâncias químicas, associado a tensões existentes no material, origina o environmental stress cracking (ESC). Estima-se que tal fenômeno seja responsável por 20 a $30 \%$ da ruptura dos diversos materiais plásticos sob tensão ${ }^{[28]}$. Basicamente, o ESC é o resultado da combinação da ação de um agente químico e de tensões externas ou residuais internas no material, originadas principalmente durante o seu processamento. O ESC pode ser explicado em termos de efeito plastificante ou do mecanismo de redução de energia superficial. Baseado na teoria da plastificação, a ruptura pode ocorrer através da concentração de tensão em um ponto originada pela difusão de pequenas moléculas do agente agressor. A teoria da redução da energia superficial sugere que um agente químico de baixa tensão superficial adere à superfície do polímero e reduz a energia total da superfície, permitindo a formação e o crescimento de poros que darão origem mais facilmente às microfissuras. $\mathrm{O}$ ESC está relacionado com a diferença no parâmetro de solubilidade entre polímero e agente químico, sendo que, quanto mais próximos esses valores, maior a probabilidade de ocorrer o fenômeno ${ }^{[29]}$. O ataque químico no material pode produzir efeitos variados, como dissolução de material polimérico, ruptura da peça ou formação de uma região translúcida ou opaca.

A propriedade de resistência à tensão pode ser 


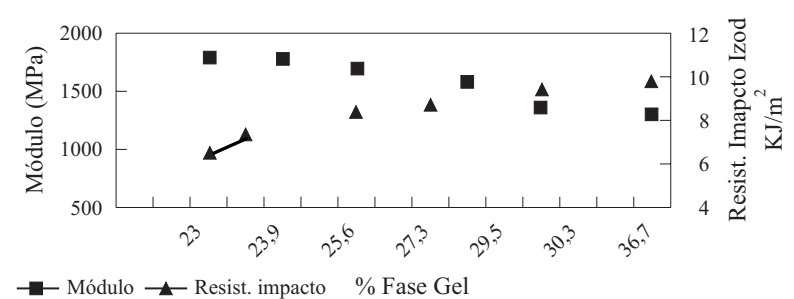

Figura 9. Variação do módulo elástico e da resistência ao impacto Izod de HIPS comercial em função do conteúdo da fase gel

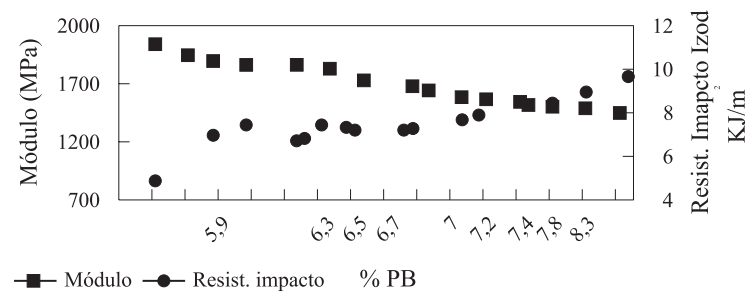

Figura 10. Variação do módulo elástico e da resistência ao impacto Izod de HIPS comercial em função do teor de PB

aperfeiçoada pela diminuição do tamanho de partículas e teor de borracha, pelo aumento do grau de entrecruzamento da borracha e pelo aumento da massa molecular do PS da matriz. Uma maior elongação pode ser obtida pelo aumento do diâmetro médio das partículas e teor de borracha, ou pela diminuição do grau de entrecruzamento da borracha.

As Figuras 9 e 10 mostram, respectivamente, a variação da resistência ao impacto Izod (entalhado) e do módulo elástico de HIPS em função do conteúdo de fase gel e do teor de borracha incorporados, de dados obtidos em ensaios de laboratório de grades comerciais de HIPS, os quais serão avaliados quanto à sua resistência química a alimentos gordurosos.

O aumento do conteúdo da fase gel permite uma maior nucleação das microfissuras, mecanismo responsável pela maior resistência ao impacto. Outra hipótese possível é que o aumento do conteúdo da fase gel seja acompanhado de um incremento no conteúdo de PS graftizado, responsável pela maior interação na interface borracha-matriz, o que consequientemente leva a um aumento da resistência ao impacto. $\mathrm{O}$ aumento do teor de borracha é responsável pela relaxação molecular, a qual dissipa parte da energia e, por isso, tem efeito sinérgico na absorção de impacto. A introdução de uma fase elastomérica na matriz rígida de PS, como esperado, promove uma diminuição no valor do módulo elástico, o que significa que o material tenacificado deforma-se em tensões menores que as verificadas para o PS homopolímero. Assim, um ganho na resistência ao impacto do material tenacificado é sempre acompanhado por uma perda no valor de seu módulo elástico.

\section{Caracterização de HIPS}

Na caracterização do HIPS, tem sido empregada a microscopia ótica ou eletrônica ${ }^{[31,35]}$, a análise química $^{[10,33,35,36]}$, a análise dinâmico-mecânica ${ }^{[30]}$ a espectroscopia de infravermelho ${ }^{[32]}$ e as técnicas de espalhamento de luz ${ }^{[37]}$, entre as mais citadas. Uma caracterização adequada deve elucidar as seguintes características do HIPS:

Morfologia

A microscopia eletrônica de transmissão (MET) tem sido largamente utilizada como forma de elucidar aspectos morfológicos e microestruturais dos materiais que são determinantes nas aplicações tecnológicas destes que requerem alto desempenho. Devido à baixa variação de densidade eletrônica entre os constituintes das misturas polímero-borracha, utiliza-se a técnica de manchamento com o tetraóxido de ósmio para se obter melhor contraste de imagem entre ambas as fases. $\mathrm{O} \mathrm{OsO}_{4}$ reage preferencialmente com as duplas ligações da borracha insaturada por reação de graftização, aumentando a diferença de densidade eletrônica entre as duas fases. $\mathrm{O}$ aumento da intensidade de espalhamento de elétrons nas regiões ricas em borracha comparadas à matriz não manchada produz um escurecimento dessas regiões na Imagem MET ${ }^{[31,35]}$. O manchamento com $\mathrm{OsO}_{4}$ fornece um excelente contraste entre as duas fases, permitindose verificar a morfologia, como a típica estrutura celular do HIPS multiple inclusion (ou salami), na qual subinclusões de PS são envolvidas por uma membrana de borracha, como mostrado na Figura 4. Em amostras de HIPS com morfologia do tipo core-shell, observa-se claramente a estrutura multicamadas das partículas de borracha, como mostrado na Figura 5.

A MET possibilita a elucidação de muitos detalhes morfológicos, particularmente quanto à estrutura interna das partículas que não são observáveis por microscopia eletrônica de varredura (MEV), que tem sido utilizada principalmente em análise de superfícies fraturadas ou microtomadas.

\section{Teor de Borracha}

Para a determinação do teor de borracha no HIPS, é utilizado um método titulométrico baseado na reação de adição de iodo na ligação dupla do PB. A titulação dos produtos da reação com tiossulfato de sódio permite a quantificação do iodo em excesso não- 
reagido e, conseqüentemente, o teor de borracha ${ }^{[10]}$. Esse método é suscetível à presença de interferentes, ou seja, todas as substâncias que tenham ligações duplas e alguns tipos de peróxidos que reagem com o iodo. Além disso, é necessário lembrar que um tipo particular de interferente deriva da polimerização, como reações de transferência de cadeia e depolimerização. Outra possibilidade de interferência é a oxidação da borracha. Esses interferentes, portanto, podem alterar o teor real de borracha no HIPS.

Outro método utilizado para a quantificação do teor de borracha é a espectroscopia de infravermelho. $\mathrm{O}$ teor de PB no HIPS pode ser medido através da medida da altura do pico de absorbância do isômero trans do PB a cerca de $965 \mathrm{~cm}^{-1}$, região que não apresenta sobreposição de bandas de absorção ${ }^{[32]}$. Esse valor é utilizado na equação da curva de calibração feita previamente com amostras-padrão. $\mathrm{Na}$ inexistência de amostras-padrão, o método titulométrico é o mais adequado.

\section{Conteúdo de Fase Gel}

Um método tipicamente usado para determinar o conteúdo (\%) da fase gel (borracha + PS graftizado e ocluído) é o teste do gel de Ruffing ${ }^{[33]}$. Nesse procedimento, o material é dissolvido em tolueno e a fase borracha é isolada por centrifugação. $O$ tolueno é removido do material isolado através de aquecimento e pressão reduzida. A relação entre a fase borrachosa seca e o peso da amostra original determina a fração em peso da segunda fase. Em uma variação deste procedimento, a amostra de HIPS é dissolvida em uma mistura de $57 \%$ de tolueno e $43 \%$ de metil etil cetona (MEK). Após centrifugação a 20.000 r.p.m., o material insolúvel é separado como um gel inchado e necessita ser lavado, no mínimo duas vezes, com solvente seco. O gel é então coagulado em etanol e seco a $40^{\circ} \mathrm{C}$. Novamente, a relação entre o peso do gel seco e a amostra original é considerada como sendo a fração em peso da segunda fase. Entretanto, os procedimentos citados anteriormente apresentam problemas. O primeiro é em relação ao solvente. É possível que as subinclusões de PS sejam extraídas das partículas em diferentes proporções por diferentes solventes. Outro problema está na centrifugação, em que a quantidade de partícula de borracha centrifugada depende do entrecruzamento da fase borracha e do tamanho de partícula: pequenas partículas são mais difíceis de centrifugar e a eficiência da centrifugação aumenta com o grau de entrecruzamento da borracha.

Essas duas dificuldades, juntamente com o fato de que as partículas podem estar parcialmente inchadas pelo solvente mesmo após a secagem, afetam a precisão das medidas da fração volumétrica das partículas ${ }^{[34-36]}$.

\section{Índice de Inchamento e Grau de Graftização}

$\mathrm{O}$ inchamento é feito, em geral, utilizando-se tolueno (após separação de fases), e a porcentagem de inchamento é calculada através da seguinte relação:

$$
\% \text { inchamento }=\mathrm{W}_{2}-\mathrm{W}_{1} / \mathrm{W}_{1} \times 100
$$

onde $\mathrm{W}_{1}$ e $\mathrm{W}_{2}$ representam o peso da amostra antes e após o inchamento, respectivamente. $\mathrm{O}$ índice de inchamento corresponde ao inverso da densidade de entrecruzamento. O grau de graftização pode ser expresso através da relação \% fase gel $/ \%$ PB. Essa medida é um tanto imprecisa, pois considera-se que o PS ocluído nas partículas de borracha esteja totalmente graftizado, o que não é verdade. Sendo assim, não há um método confiável e reprodutível para que se quantifique corretamente o grau de graftização no $\operatorname{HIPS}^{[35]}$.

\section{Tamanho de Partícula}

Atualmente, o principal método de medida do tamanho de partícula é a técnica de espalhamento de luz. A difração de uma radiação tipo laser é colhida para pequenos ângulos de espalhamento e, através de um tratamento matemático via matrizes (software), é feita uma medida do tamanho das partículas, levando-se em conta as suas três dimensões ${ }^{[37]}$. O resultado é relacionado com a contribuição da freqüência de cada tamanho de partícula. Dessa forma, obtémse também uma curva de distribuição de tamanhos de partícula. Essa medida ainda pode ser feita através de técnicas microscópicas, porém a medida é feita em somente duas dimensões ${ }^{[35]}$.

\section{Aplicações do HIPS}

As resinas comerciais de HIPS têm sido usadas tanto em processos de moldagem por injeção como por extrusão. Geralmente, as resinas que apresentam baixa viscosidade do fundido são usadas para aplicações em moldagem por injeção, e as resinas com alta 
viscosidade do fundido são mais apropriadas para aplicações em extrusão. Outra aplicação que vem ganhando espaço é na termoformagem, principalmente na indústria de embalagens para alimentos e, em fase incipiente, na moldagem de peças maiores, como gabinetes para geladeiras.

O HIPS, por sua maior resistência ao impacto, é usado em peças de maior exigência física em relação ao PS não-tenacificado. É usado em artigos industriais, como peças de máquinas e veículos, caixas para rádio, televisão e microcomputadores, grades de ar condicionado, peças internas e externas de aparelhos eletrônicos, de telecomunicações, toca-fitas de carro, cabos e armações para guarda-chuvas, gabinetes para geladeira. Também é utilizado na indústria calçadista (saltos) e na de embalagens de proteção contra choque, bem como em artigos de linha, como para cozinha e higiene, além de pratos, talheres, copos de café, água, refrigerantes e iogurte, utensílios diversos como brinquedos, jogos e artigo descartáveis ${ }^{[3]}$.

\section{Comentários Gerais}

Apesar de ter sido um material alvo de diversos estudos nos últimos anos, o HIPS ainda possui algumas características que são importantes com relação às suas propriedades e à sua aplicação que devem ser melhor elucidadas. Entre elas, encontra-se a necessidade de uma melhor distinção entre os conceitos de conteúdo de fase gel e fração volumétrica da segunda fase, que em uma primeira análise pode parecer apenas uma questão de conversão densidade-volume entre os constituintes, mas que, na realidade, é uma questão mais complexa. Outra lacuna na tecnologia de tenacificação do PS é a correta quantificação do índice de graftização e o desenvolvimento de métodos mais eficientes e reprodutíveis que a técnica gravimétrica (método de Ruffing ${ }^{[33]}$ ) na caracterização da fase borracha.

Para que se ultrapasse a atual fronteira de conhecimento, com relação à tenacificação do PS com elastômeros de tipo SBR e PB, a incorporação de novos tipos de borracha na matriz de PS, principalmente os elastômeros termoplásticos à base de estireno disponíveis no mercado, poderá ser uma nova opção na obtenção de novos grades de HIPS.

\section{Agradecimentos}

Os autores agradecem ao $\mathrm{CNPq}$ pela bolsa de mestrado e à INNOVA S.A. pelas amostras de HIPS e pelo apoio analítico.

\section{Referências Bibliográficas}

1. Bucknall, C. B. - "Toughened Plastics",Applied Science, Londres (1977).

2. Echte, A. - "Rubber Toughened Plastics", C. K. Riew (ed.), American Chemical Society, Washington (1989).

3. Soderquist, M. E. \& Dion, R. P. - "Encyclopedia of Polymer Science and Engineering", vol. 16, pg. 88-97, segunda edição (1989).

4. Cook, D. G.; Rudin, A. \& Plumtree, A. - J. of Appl. Polym. Sci., 48, p.75 (1993).

5. Ostromislensky, I. I. - Patente Americana 1,613,673 (1927).

6. Mui, E. T. C.; Boateng, V. B.; Fellers, J. F. \& White, J. L. - J. of Appl. Polym. Sci., 27, p.1395 (1982).

7. Peng, F. M. - J. of Appl. Polym. Sci 40, p.1289 (1990).

8. Gupta, V. K. ; Bhargava, G. S. \& Bhattacharyya, K. K. J. Macromol. Sci.-Chem., A16, p. 1107 (1981)

9. Choi, J. H.; Ahn, K. H. \& Kim, S. Y. - Polymer, 41, p.5229 (2000).

10. Boletim Técnico, "Innova-Etilbenzeno, Estireno, Poliestireno" (1998).

11. Katime, I.; Quintana, J. R. \& Price, C. - Mat. Lett., 22, p.297 (1995).

12. Bubeck, R. A.; Buckley, D. J.; Kramer, E. J. \& Brown, H. R. - J. of Mat. Sci., 26, p.6249 (1991).

13. Gilbert, D. G. \& Donald, A. M. - J. of Mat. Sci., 21, p.1819 (1986).

14. Magalhães, A. M. L. \& Borggreve, R. J. M. - Macromolecules, 28, p.5841 (1995).

15. Donald, A. M. \& Kramer, E. J. - J. of Appl. Polym. Sci., 27, p.3729 (1982).

16. Dagli, G.; Argon, A. S. \& Cohen, R. E. - Polymer, 36, p.2173 (1995).

17. Piorkowska, E.; Argon, A. S. \& Cohen, R. E. - Macromolecules, 23, p.3838 (1990).

18. Keskkula, H.; Schwars, M. \& Paul, D. R. - Polymer, 27, p.211 (1986).

19. Dompas, D. \& Groeninckx, G. - Polymer, 35, p.4743 (1994). 
20. Bubeck, R. A.; Buckley, D. J.; Kramer, E. J. \& Brown, H. R. - J. of Mat. Sci., 26, p.6249 (1991).

21. Cigna, G.; Maestrini, C.; Castellani, L. \& Lomellini, P. -J. of Appl. Polym. Sci., 44, p.505 (1992).

22. Maestrini, C.; Callaioli, A.; Castellani, L. \& Ferrando, A. - J. of Mat. Sci., 34, p.6045 (1999).

23. Cigna, G.; Lomellini, P. \& Merlotti, M. - J. of Appl. Polym. Sci., 37, p.1527 (1989).

24. Siberberg, J. \& Han, C. D. - J. of Appl. Polym. Sci., 22, p.599 (1978).

25. Okamoto, Y.; Miyagi, H.; Kakugo, M. \& Takahashi, K. - Macromolecules, 24, p.5639 (1991).

26. Maestrini, C.; Pisoni, K. \& Kaush, H. H. - J. of Mat. Sci., 31, p.3249 (1996).

27. Cho, K.; Lee, M. S. \& Park, C. E. - Polymer, 38, p.4641 (1997).

28. Hough, M. C. \& Wright, D. C. - Polymer Testing, 15, p.407 (1996).
29. Arnold, J. C. - Mat. Sci. and Eng., A197, p.119 (1995).

30. Sardelis, K.; Michels, H. J. \& Allen, G.- J. of Appl. Polym. Sci., 28, p.3255 (1983).

31. Correa, C. A. - Polímeros: Ciência e Tecnologia - Jan/ Mar, p.24 (1995).

32. Lacoste, J.; Delor, F.; Pilichowski, J. F.; Singh, R. P.; Prasad, A. V. \& Sivaram, S. - J. of Appl. Polym. Sci., 59, p.953 (1996).

33. Ruffing, N. R. - Patente Americana 3,243,481 (1966).

34. Hall, R. A. -J. of Mat. Sci., 25, p.183 (1990).

35. Maestrini, C.; Merlotti, M.; Vighi, M. \& Malaguti, E. J. of Mat. Sci., 27, p.5994 (1992).

36. Alzandi, S.; Bonifaci, L.; Malaguti, E.; Vighi, M. \& Ravanetti, G. P. - J. of Mat. Sci. Lett., 13, p.1555 (1994).

37. Salomons, G. J.; Singh, M. A.; Bardouille, T. \& Foran, W. A. - Macromolecules, 32, p.1264 (1999).

Recebido: $25 / 05 / 01$

Aprovado: 04/10/01 